\title{
Editorial
}

Dermatology

Published online: June 18, 2009

DOI: $10.1159 / 000225933$

\section{Role of Artificial Neural Networks in Dermatology}

\author{
J.-M. Renders ${ }^{\mathrm{a}} \quad$ T. Simonart ${ }^{\mathrm{b}}$ \\ ${ }^{a}$ Xerox Research Centre Europe, Meylan, France; ${ }^{b}$ Department of Dermatology, Hôpital Universitaire Erasme, \\ Brussels, Belgium
}

\section{Key Words}

Artificial intelligence - Melanoma - Neural networks • Vitiligo

Pattern recognition is a branch of computer science and artificial intelligence capable of analyzing complex medical data. Its potential to exploit meaningful relationships within a data set can be used in the diagnosis, treatment and outcome prediction in many clinical scenarios. Among the different approaches of pattern recognition, the connectionist one, mainly materialized by artificial neural networks (ANNs) [1,2], is constructed to roughly simulate processes of the central nervous system in the human brain. An ANN consists of a set of processing units (nodes) that simulate neurons and are interconnected via a set of 'weights' (analogous to synaptic connections in the nervous system) in a way that allows signals to propagate through the network in parallel. The nodes (neurons) are simple computing elements. They accumulate inputs from other neurons by means of a weighted sum. If a certain threshold is reached, the neuron sends information to all other connected neurons otherwise it remains quiescent, or, most often, the neuron can simply transform the weighted sum by some sigmoid function that softens this binary decision mechanism (the nonlinearity of the sigmoid function is a key point). One major difference with respect to traditional hand-coded rule- based systems is the learning capacity of an ANN. At the very beginning of a training process, an ANN contains no explicit information. Then a large number of cases with a known outcome are presented to the system, and the weights of the interneuronal connections are changed by a training algorithm designed to minimize the total error of the system [1-3]. ANNs constitute a digitized model of a biological brain and can detect complex nonlinear relationships between variables in data that the human brain may fail to detect [4]. Furthermore an ANN can be used when the influencing factors on a certain event are not exactly known as it is the case in financial or weather forecasts.

Although ANNs have been developed in the last few decades for many different applications in medical science and in biomedical research (e.g. drug design, pharmacoepidemiology, diagnosis and prognosis in clinical medicine) [4-6], their use in the field of dermatology remains relatively limited.

So far, the main use of ANNs in dermatology is the in vivo differentiation of benign versus malignant pigmented lesions. Various computational vision-based diagnostic systems employ ANN-based algorithms to extract discriminative features from images of pigmented skin lesions and to provide an assessment of malignancy risk. The works using ANN-based analysis of pigmented skin lesions have shown variable results and mainly emphasize the need for further validation before the malignan-

\section{KARGER \\ Fax +4161306 1234 \\ E-Mail karger@karger.ch \\ www.karger.com}

(C) 2009 S. Karger AG, Basel

$1018-8665 / 09 / 2192-0102 \$ 26.00 / 0$

Accessible online at: www.karger.com/drm
Dr. T. Simonart

Department of Dermatology, Hôpital Universitaire Erasme 808 , route de Lennik

BE-1070 Brussels (Belgium)

Tel. +32 255546 12, Fax +32 255549 69, E-Mail tsimonar@ulb.ac.be 
cy risk assessment features can be widely used in clinical practice [7-11]. Still, there is evidence that ANNs can be trained to adequately discriminate between benign and malignant pigmented skin lesions. Preliminary data suggest that ANN-based analysis of electric impedance may also be a promising useful tool for the identification of nonmelanoma skin cancers [12].

Because ANNs can easily consider variable interactions and create a nonlinear prediction model, they offer a more flexible prediction of risk assessment and prognosis of cancer than traditional methods (e.g. linear or logistic regression, linear discriminant analysis). Again, relatively few data are available in the field of dermatology. In 2005, Mian et al. [13] reported that ANN-based analysis of serum proteomic fingerprinting could be a valuable tool for identifying high-risk melanoma patients.

ANNs have also been used in predictive models for allergic diseases, such as atopic dermatitis, and have been shown to provide a better predictive performance than logistic regression models $[14,15]$. Another application of ANNs in allergic diseases is the prediction of the risk level of pollen in the air, using previous pollen and meteorological information [16].

In this issue of Dermatology, Cazzaniga et al. [17] used an ANN-based approach to anticipate treatment duration in vitiligo patients treated with excimer laser therapy.

In the machine learning community, using neural networks to perform nonlinear regression or classification is a countertrend (at least till 2006). One reason of this relative abandon lies in the following fact: this family of methods generated so many abuses in the late 90s that most scientific communities came back to more principled ways of solving the task. That gave rise to new approaches (such as extensions of lasso regression [18], support vector machines and kernel methods [19]) that are more transparent and easier to motivate, while achieving superior performance with respect to ANN-based approaches.

The main point is around 'model regularization', i.e. how to constrain the model to have better generalization properties or, in other words, less overfitting, by better controlling the complexity of the models and the associated error bounds. This is the price to be paid for having a lot of degrees of freedom in both the architecture (number of hidden layers and neurons, transfer function, ...) and in the training parameters (learning rate, number of iterations and epochs, ...). On the one hand, modelers using ANNs have multiple sources of flexibility to better capture complex nonlinear relationships in their data but, in contrast, they have to take serious precautions in order to avoid overfitting and poor prediction capabilities on new data. One important requirement is to use a huge amount of training data in order to be able to identify the model parameters with statistical significance (a standard rule of thumb is to have at least 10 observations for 1 parameter tuned) but this is not sufficient: training data must be representative of all possible operational conditions and cover the space of inputs as it will be in future test conditions. As ANNs have much more parameters to tune than traditional models, they consequently need much more training data. In practice, as training data are not as plentiful as required, a suitable 'model regularization' scheme is needed. Model regularization was the weak point of neural networks, and this may explain why they practically failed in a huge number of applications in the late 90s. Indeed, many available ANN softwares did not provide the user with some 'default' efficient regularization mechanisms, simply because it is not possible to automatically and optimally adjust the regularization settings for every problem; many researchers underestimated the importance of the regularization process, and this resulted in relatively poor performance in practical applications.

On the other side, contrarily (or in reaction) to ANNs, the main trend up to very recently was to separate the feature selection/extraction/construction problems from the learning problem itself, in order to gain in generality and to better control each of these two fundamental steps by trying to separate their effects on the global performance. Kernel methods [19], for instance, gave rise to pattern recognition algorithms able to deal very efficiently and very robustly with nonlinear regressors, even with infinite dimensions. Once a kernel function has been chosen that implicitly defines a suitable feature space (a kernel is nothing else than some well-defined similarity measure in this feature space), the learning algorithms usually involve very few parameters to tune (typically, only 1 regularization parameter that controls the overfitting risk). This offers an interesting contrast to ANNs. Moreover, the choice of a kernel function is in general an easier task than parameter tuning in ANNs and, in general, allows the modeler to introduce prior knowledge in a principled way.

These recent developments in machine learning have as a consequence the renewed interest in ANNs but, this time, adopting a more principled viewpoint, by taking all necessary precautions to control the model regularization issue [20] (the modern view of the well-known 'bias- 
variance' problem [21]) as well as introducing as much prior knowledge as possible by constraining the network architecture and by explicitly constructing/selecting ad hoc features instead of letting the ANN try to extract them itself; one particularly good example of these new developments lies in what is now called 'deep architectures' [22], the direct offspring of ANNs.
These are the major challenges that, even in the field of quantitative dermatology, the researchers and practitioners have to face when using ANN-based technology or tools. Thus, although artificial intelligence techniques have the potential to be applied in various fields of dermatology, there is a need for further appropriately designed clinical trials before these emergent techniques can be widely used in the clinical practice.

\section{References}

1 Bishop CM: Neural Networks for Pattern Recognition. Oxford, Oxford University Press, 1995.

2 Ripley BD: Pattern Recognition and Neural Networks. Cambridge, Cambridge University Press, 1996.

>3 Traeger M, Eberhart A, Geldner G, Morin AM, Putzke C, Wulf H, Eberhart LH: Artificial neural networks: theory and applications in anesthesia, intensive care and emergency medicine. Anaesthesist 2003;52: 1055-1061.

4 Patel JL, Goyal RK: Applications of artificial neural networks in medical science. Curr Clin Pharmacol 2007;2:217-226.

$\checkmark 5$ Ramesh AN, Kambhampati C, Monson JR, Drew PJ: Artificial intelligence in medicine. Ann R Coll Surg Engl 2004;86:334-338.

$\checkmark 6$ Hanai T, Honda H: Application of knowledge information processing methods to biochemical engineering, biomedical and bioinformatics fields. Adv Biochem Eng Biotechnol 2004;91:51-73.

7 Binder M, Steiner A, Schwarz M, Knollmayer S, Wolff K, Pehamberger H: Application of an artificial neural network in epiluminescence microscopy pattern analysis of pigmented skin lesions: a pilot study. Br J Dermatol 1994;130:460-465.

$>8$ Barzegari M, Ghaninezhad H, Mansoori P, Taheri A, Naraghi ZS, Asgari M: Computeraided dermoscopy for diagnosis of melanoma. BMC Dermatol 2005;5:8.
$>9$ Boldrick JC, Layton CJ, Nguyen J, Swetter SM: Evaluation of digital dermoscopy in a pigmented lesion clinic: clinician versus computer assessment of malignancy risk. J Am Acad Dermatol 2007;56:417-421.

10 Carrara M, Bono A, Bartoli C, Colombo A, Lualdi M, Moglia D, Santoro N, Tolomio E, Tomatis S, Tragni G, Santinami M, Marchesini R: Multispectral imaging and artificial neural network: mimicking the management decision of the clinician facing pigmented skin lesions. Phys Med Biol 2007;52: 2599-2613.

11 Surowka G, Grzesiak-Kopec K: Different learning paradigms for the classification of melanoid skin lesions using wavelets. Conf Proc IEEE Eng Med Biol Soc 2007;2007: 3136-3139.

12 Dua R, Beetner DG, Stoecker WV, Wunsch DC 2nd: Detection of basal cell carcinoma using electrical impedance and neural networks. IEEE Trans Biomed Eng 2004;51:6671.

13 Mian S, Ugurel S, Parkinson E, Schlenzka I, Dryden I, Lancashire L, Ball G, Creaser C, Rees R, Schadendorf D: Serum proteomic fingerprinting discriminates between clinical stages and predicts disease progression in melanoma patients. J Clin Oncol 2005;23: 5088-5093.

14 Takahashi K, Hayasawa H, Tomita M: A predictive model for effect of atopic dermatitis in infancy by neural network and multiple logistic regression (in Japanese). Arerugi 1999;48:1222-1229.
15 Tomida S, Hanai T, Koma N, Suzuki Y, Kobayashi T, Honda $\mathrm{H}$ : Artificial neural network predictive model for allergic disease using single nucleotide polymorphisms data. J Biosci Bioeng 2002;93:470-478.

16 Castellano-Méndez M, Aira MJ, Iglesias I, Jato V, González-Manteiga W: Artificial neural networks as a useful tool to predict the risk level of Betula pollen in the air. Int J Biometeorol 2005;49:310-316.

17 Cazzaniga S, Sassi F, Mercuri SR, Naldi L: Prediction of clinical response to excimer laser treatment in vitiligo by using neural network models. Dermatology 2009;219: 133-137.

18 Hastie T, Tibshirani R, Friedman JH: The Elements of Statistical Learning: Data Mining, Inference, and Prediction. New York, Springer, 2001.

19 Shawe-Taylor J, Cristianini N: Kernel Methods for Pattern Analysis. Cambridge, Cambridge University Press, 2004.

20 Kwok T, Yeung D: Bayesian regularization in constructive neural networks; in von der Malsburg C, von Seelen W, Vorbrüggen JC, Sendhoff B (eds): Lecture Notes in Computer Science. Heidelberg, Springer, 1996, pp 557562.

21 Geman S, Bienenstock E, Doursat R: Neural networks and the bias/variance dilemma. Neural Comput 1992;4:1-58.

22 Bengio Y: Learning deep architectures for AI; in Bengio Y (ed): Foundations and Trends in Machine Learning. 2009, in press. 